\title{
A portable cost-effective amplitude and phase antenna measurement system
}

\author{
Yuri Álvarez López, Senior Member, IEEE, María García-Fernández, Student Member, IEEE, \\ Fernando Las-Heras, Senior Member, IEEE
}

\begin{abstract}
This contribution presents a simple, cost-effective system for complex measurements of the field radiated by an antenna. In particular, the proposed architecture is based on an array of antenna probes connected to a set of phase difference detectors and a power detector. Phase difference measurements are processed to recover the phase of the antenna under test. The main novelty of the proposed system is that it does not require regular sampling, making it suitable for in-situ antenna measurements. The influence of parameters such as the number of antennas composing the array (and hence the number of phase difference detectors) and the positioning errors is analyzed. The proposed architecture, implemented with off-the-shelf hardware, has been assessed for antenna measurement and diagnostics. A discussion of the issues related to the implementation of the system has been included, as well as the proposed solutions to improve its accuracy. Finally, diagnostics and radiation pattern measurement results are compared with those calculated with other phaseless techniques.
\end{abstract}

Index Terms-Electromagnetic fields, Antenna measurement, Phaseless techniques, Antenna diagnostics.

\section{INTRODUCTION}

A NTENNA characterization is one of the key steps in the development of modern communications systems [1]. Traditionally, this procedure has been conducted in dedicated facilities such as anechoic chambers [2]. However, improvements in the accuracy and miniaturization of radiofrequency components together with novel and precise positioning systems have resulted in novel, portable systems for in-situ antenna characterization. For example, in [3] a handheld system is proposed for manually acquiring near field samples, taking advantage of millimeter-accuracy optical positioning systems. [4],[5] present airborne-based antenna

Manuscript received September 25, 2019. This research has been funded by the Ministerio de Educación - Gobierno de España under Grant FPU15/06341; by the Ministerio de Ciencia, Innovación y Universidades Gobierno de España under Project RTI2018-095825-B-I00 ("MilliHand"); by the Government of the Principality of Asturias (PCTI) and European Union (FEDER) under Grant IDI/2018/000191; by the Instituto Universitario de Investigación Industrial de Asturias (IUTA) under Project SV-19-GIJON-1-17 ("RadioUAV"); and by the University of Oviedo, under project PAPI-18-GR2010-0015.

Y. Álvarez López, M. García-Fernández, and F. Las-Heras are with the Área de Teoría de la Señal y Comunicaciones, Universidad de Oviedo. Edificio Polivalente, Módulo 8, Campus Universitario de Gijón. E-33203, Gijón (Spain). Email: alvarezyuri@uniovi.es. Phone: +34985182281. Fax: +34985182466 . measurement systems, which are able to provide antenna diagnostics without affecting the normal operation of the antenna. The impact of the radiofrequency circuitry in the antenna performance can be assessed by means of on-wafer measurement techniques [6].

In general, antenna measurement requires the acquisition of both amplitude and phase (that is, complex measurements of the radiated field) to enable antenna diagnostics (detection of malfunctioning elements) [7] and a near field (NF) to far field (FF) transformation in case the field was measured in the NF region of the Antenna Under Test (AUT). However, phase measurement is more complex than amplitude-only acquisition, requiring high accuracy and cost hardware. Besides, a physical connection between the AUT and the measurement equipment is needed for complex acquisitions in order to have a phase reference. However, having physical access to the AUT and/or the transmitter can be difficult in the case of on-site antenna measurements, and it also requires temporary interruption of the communications system served by the AUT.

TABLE I

Phase ACQuisition TeChNiques in AnTENnA MEASurement

\begin{tabular}{|c|c|c|}
\hline $\begin{array}{l}\text { Group of } \\
\text { methods }\end{array}$ & $\begin{array}{l}\text { Refe- } \\
\text { rence }\end{array}$ & Description / main feature \\
\hline \multirow{3}{*}{$\begin{array}{l}\text { Interfero- } \\
\text { metric or } \\
\text { holographic } \\
\text { techniques }\end{array}$} & $\begin{array}{l}{[8],} \\
{[9]}\end{array}$ & $\begin{array}{l}\text { Use of a reference antenna or a reference } \\
\text { signal. }\end{array}$ \\
\hline & {$[10]$} & Synthetic reference. \\
\hline & [11] & $\begin{array}{l}\text { Phase retrieval based on frequency domain } \\
\text { techniques. Suitable for broadband antennas. }\end{array}$ \\
\hline \multirow{3}{*}{$\begin{array}{l}\text { Iterative } \\
\text { phase } \\
\text { retrieval } \\
\text { methods }\end{array}$} & [12] & $\begin{array}{l}\text { Based on forward-backward techniques. } \\
\text { Planar and polar domains. }\end{array}$ \\
\hline & $\begin{array}{l}{[13],} \\
{[14]}\end{array}$ & $\begin{array}{l}\text { Based on non-linear cost function } \\
\text { minimization. Works with arbitrary surfaces. }\end{array}$ \\
\hline & [15] & $\begin{array}{l}\text { Wave mode expansion-based formulation for } \\
\text { phaseless NF measurements. }\end{array}$ \\
\hline \multirow{3}{*}{$\begin{array}{l}\text { Phase } \\
\text { difference }\end{array}$} & {$[16]$} & $\begin{array}{l}\text { Broadband technique. Requires equally- } \\
\text { spaced sampling. }\end{array}$ \\
\hline & {$[17]$} & $\begin{array}{l}\text { Hybrid phase difference-interferometry } \\
\text { technique. }\end{array}$ \\
\hline & $\mathrm{a}$ & $\begin{array}{l}\text { Capable of working with non-regular } \\
\text { sampling. Tested for cuts only. }\end{array}$ \\
\hline
\end{tabular}

This contribution.

Thus, antenna measurement systems can be simplified by indirect measurement of the phase, also avoiding the transmitter and receiver to be phase-locked (which is impractical for in-situ antenna measurements). For this goal, several phase retrieval techniques have been developed in the 
last decades. They can be classified in three main groups, as listed in Table 1.

Interferometric or holographic techniques [8]-[11] are based on the knowledge of a reference source (e.g. a reference measurement or a synthetic reference). They have proved to be quite accurate and fast in terms of measurement time, but at the expense of increasing hardware complexity.

Iterative phase retrieval techniques [12]-[15] require measurements of the amplitude of the field radiated by the AUT at two or more acquisition surfaces. The main advantage is hardware simplicity (just a single power detector is needed). However, the measurement and processing time is increased, and there is also some risk of stagnation of the iterative solvers since they usually consist of minimizing a non-linear costfunction. Convergence of these iterative solvers is conditioned by the position of the acquisition surfaces as well as the choice of the initial guess [14].

There is a third group of methods for indirect phase acquisition, based on amplitude and phase difference detectors [16],[17]. Briefly, the idea is to measure the phase difference at, at least, two receiving antennas. If all the distances in the problem are known (the position of the AUT and the position of the two receiving antennas), then the phase function can be retrieved by combining (or stitching) multiple phase difference measurements (except for a phase reference constant value).

This contribution focuses on the last group of techniques, aiming to continue developing simple, low-cost antenna measurement systems for in-situ measurements. In particular, the goal is to develop and test a novel architecture of a receiver capable of providing amplitude and phase information with a single acquisition, overcoming the limitations of iterative phase retrieval techniques and interferometric methods. Compared to previous works, the major contribution is that the need for regular sampling is avoided, provided that the Nyquist sampling rate is fulfilled.

\section{Methodology}

\section{A. System architecture}

The proposed architecture for the phase acquisition system based on phase difference measurements is depicted in Fig. 1. The output of an array of $N$ probe antennas is connected to $N-1$ phase difference detectors. Each detector provides a voltage proportional to the difference of the phase of the acquired field between antennas $\mathrm{n}$ and $\mathrm{n}+1, \Delta \Phi_{\mathrm{n}, \mathrm{n}+1}$. The amplitude of the measured field, $|E|$, is acquired by means of a conventional power detector. It can be acquired either at the output of each antenna (e.g. by placing a 3-port power splitter), or at the output of one of the antennas (as in Fig. 1), assuming slow spatial variation of the amplitude of the field radiated by the AUT.

This configuration is intended for measurements along one dimension (e.g. measurement of the main cuts of the AUT), although it can be expanded for two-dimensional radiated field measurements. The array is moved in front of the AUT at a given distance $\boldsymbol{r}(x, y)$. At each $m$-th measurement position $\boldsymbol{r}_{\mathrm{m}}\left(x_{\mathrm{m}}, y_{\mathrm{m}}\right)$, the amplitude $|\mathrm{E}|_{\mathrm{m}}$ and the phase differences $\left[\Delta \Phi_{1,2}, \Delta \Phi_{2,3}, \ldots, \Delta \Phi_{\mathrm{N}-1, \mathrm{~N}}\right]_{\mathrm{m}}$ are recorded. It must be remarked that measurement positions can be arbitrarily placed, provided that the distance between two consecutive measurement positions satisfies (1):

$$
\left\|r_{m}\left(x_{m}, y_{m}\right)-r_{m+1}\left(x_{m+1}, y_{m+1}\right)\right\|<\lambda / 2
$$

This means that no uniform sampling is required, but there must be partial overlap between two consecutive positions, $m$ and $m+1$, as depicted in Fig. 2 .

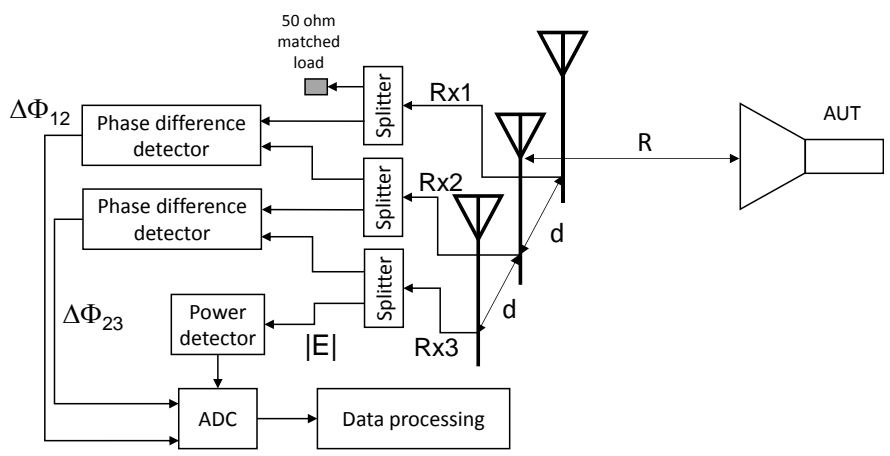

Fig. 1. Architecture of the proposed system for phase acquisition based on phase difference measurement. In this example, three antennas are considered $(N=3)$.
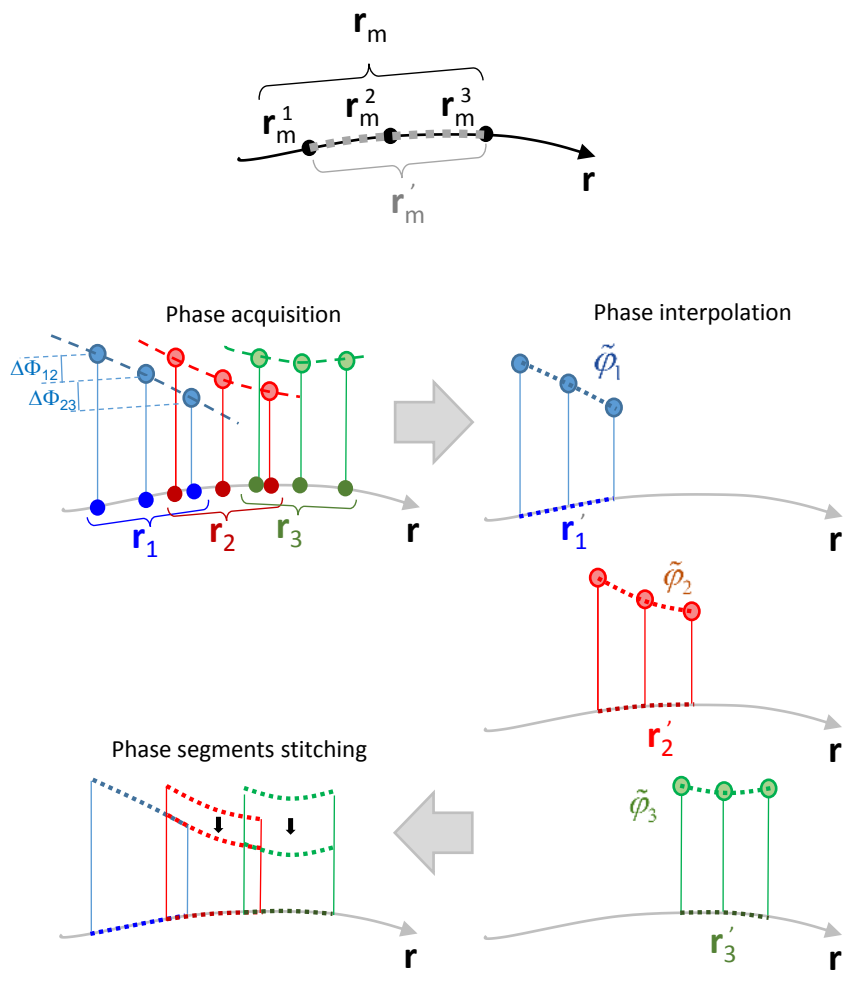

Fig. 2. Processing of the acquired phase differences at each $m$-th measurement position, and stitching of the resulting phase segments.

\section{B. Data processing}

Next step is the recovery of the phase of the measured field from the acquired phase difference at each $m$-th measurement position. First, the set of $\left[\Delta \Phi_{1,2}, \Delta \Phi_{2,3}, \ldots, \Delta \Phi_{\mathrm{N}-1, \mathrm{~N}}\right]_{\mathrm{m}}$ phase 
differences is adjusted according to a polynomial function pol (dashed lines depicted in Fig. 2), as outlined in Eq. (2). This polynomial function is used to estimate the phase along a segment of the measurement path, $\boldsymbol{r}_{m}^{\prime}\left(x_{m}^{\prime}, y_{m}^{\prime}\right), \boldsymbol{r}_{m}^{\prime} \subseteq \boldsymbol{r}_{m}$. Different phase segments $\tilde{\varphi}_{m}\left(r_{m}^{\prime}\right)$ can be stitched by adjusting the phase reference of each $m$-th segment (which is set to zero initially, $\varphi^{1}=0^{\circ}$ ). In Eq. (2), $P$ is the order of the polynomial function, whose value is conditioned by the number of elements of the array, $N$. polyfit and polyval functions denote the fitting and evaluation operations.

$$
\begin{aligned}
& \text { pol }=\operatorname{polyfit}\left(\left[x_{m}^{1}, x_{m}^{2}, \ldots, x_{m}^{N}\right],\left[y_{m}^{1}, y_{m}^{2}, \ldots, y_{m}^{N}\right],\right. \\
& \left.\left[\varphi^{1}, \varphi^{1}+\Delta \Phi_{m}^{1,2}, \ldots, \varphi^{1}+\Delta \Phi_{m}^{1,2}+\ldots+\Delta \Phi_{m}^{N-1, N}\right], P\right) \\
& \tilde{\varphi}_{m}=\operatorname{polyval}\left(\operatorname{pol}\left(r_{m}^{\prime}\left(x_{m}^{\prime}, y_{m}^{\prime}\right)\right)\right)
\end{aligned}
$$

Once the phase at each segment of the measurement path has been estimated, a stitching operation is conducted to combine all the segments avoiding discontinuities, as outlined in Fig. 2.

$$
\begin{aligned}
& \tilde{\varphi}(r(x, y))=\operatorname{stitching}\left(\tilde{\varphi}_{1}\left(r_{1}^{\prime}\right), \tilde{\varphi}_{2}\left(r_{2}^{\prime}\right), \ldots, \tilde{\varphi}_{M}\left(r_{M}^{\prime}\right)\right), \\
& r_{1}^{\prime} \cup r_{2}^{\prime} \cup \ldots \cup r_{M}^{\prime}=r
\end{aligned}
$$

The result of the stitching operation is the phase along the measurement path, $\boldsymbol{r}(x, y)$. The first segment used in the stitching operation will set the $0^{\circ}$ constant phase reference.

\section{ANALYSIS OF THE PROPOSED SYSTEM}

The proposed methodology for phase acquisition based on phase difference measurements can be sensitive to: electromagnetic noise and interferences existing in the measurement setup, noise introduced by the power detector and the phase difference detectors, and positioning and georeferring errors. Besides, the number of elements of the antenna array may impact the performance of the system. Finally, coupling between the elements of the array used as probe can distort the measurements. The influence of these parameters is analyzed in this section, presenting two examples for linear (one-dimensional, 1D) and planar (two-dimensional, 2D) acquisitions. These examples are based on antenna models obtained from measurements at spherical range in anechoic chamber. The flowchart of the analysis followed is depicted in Fig. 3.

\section{A. One-dimensional case}

For the 1D analysis, a base station antenna working at 1800 $\mathrm{MHz}$ is selected as AUT. This antenna was measured at spherical range in anechoic chamber and characterized using an equivalent currents model [13]. The reason why this AUT has been chosen is because its radiation pattern exhibits multiple sidelobes, so it is a challenging model for antenna measurement techniques testing and benchmarking. From this model, the NF radiated at $D=4 \mathrm{~m}$ along a straight segment of $L=10 \mathrm{~m}$ has been assessed, introducing positioning and georeferring errors. For this configuration, the FF angular margin of validity is $\theta_{\mathrm{FF}}= \pm 47^{\circ}$. A Signal-to-Noise Ratio (SNR) of 40 $\mathrm{dB}$, which is a typical value for non-fully anechoic environments, has been considered.

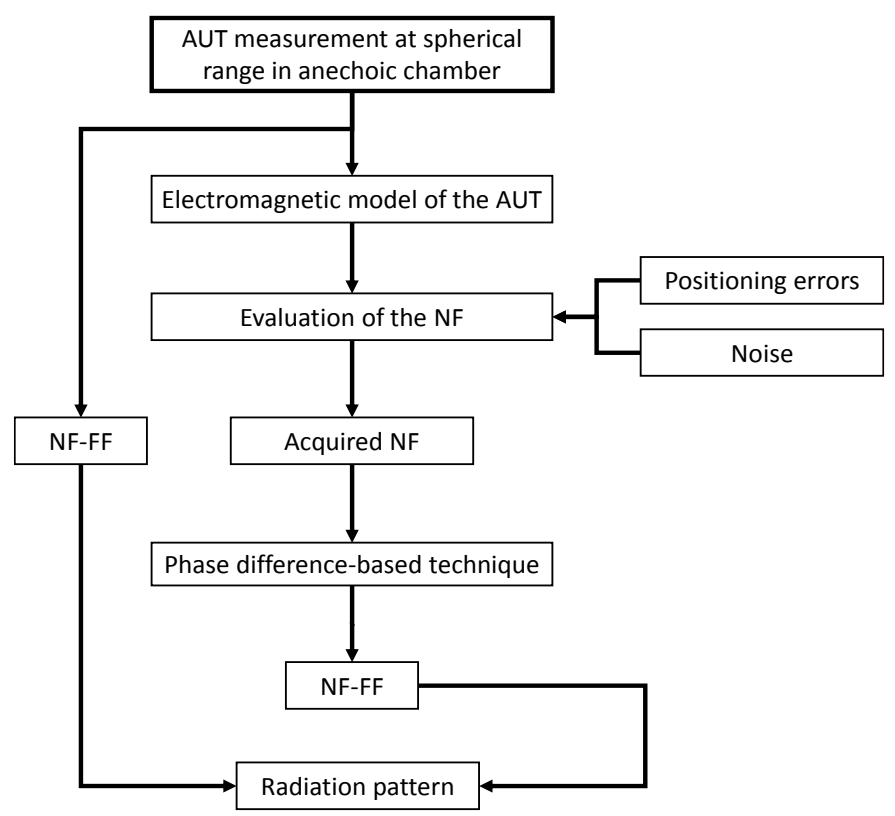

Fig. 3. Flowchart of the methodology followed for the analysis of the proposed phase difference-based technique.

An $N$-element array of isotropic antennas has been considered as probe, with $N$ ranging from 2 to 4 . Spacing between antenna array elements is $d=5 \mathrm{~cm}(0.3 \lambda)$, to ensure proper sampling of the NF phase. Spacing between consecutive measurement positions is $\Delta y=3 \mathrm{~cm}+d(N-2)$, to ensure overlap.

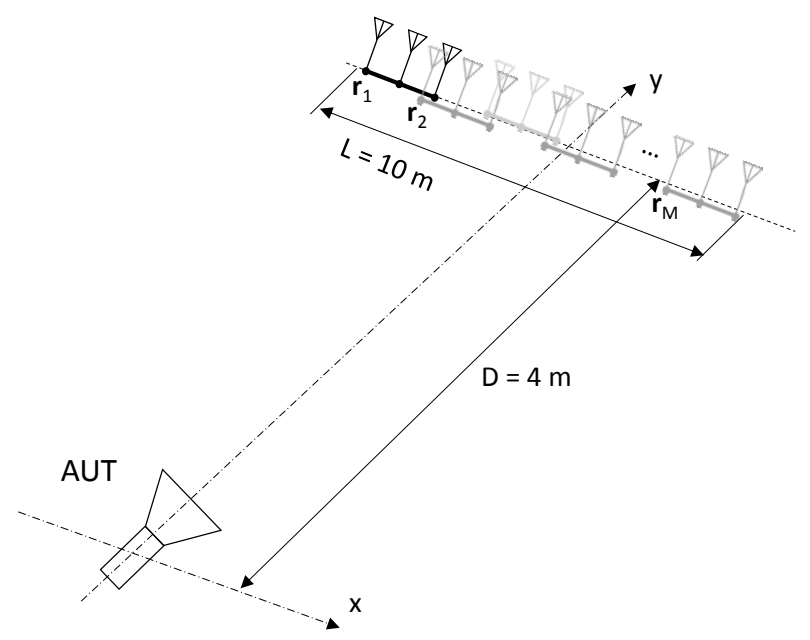

Fig. 4. NF acquisition setup for the analysis of the proposed technique.

First, the NF is acquired in the domain depicted in Fig. 4 considering there are no positioning errors in the array. Reconstructed phases for $N=2, N=3$, and $N=4$ elements arrays are depicted in Fig. 5 (a), together with the reference phase, which is the phase of the NF directly calculated at the acquisition positions.

The phase error $\varepsilon_{\varphi}$ is defined as follows (5): 


$$
\begin{aligned}
& \mathcal{E}_{\varphi}= \\
& \operatorname{wrap}\left(\operatorname{unwrap}\left(\tilde{\varphi}(r)-\tilde{\varphi}\left(r_{\max }\right)\right)-\operatorname{unwrap}\left(\varphi(r)-\varphi\left(r_{\max }\right)\right)\right)
\end{aligned}
$$

where wrap and unwrap denote phase wrapping and unwrapping operations. $\boldsymbol{r}_{\max }$ is the position where the maximum of the measured amplitude, $|\mathrm{E}|$, is recorded.
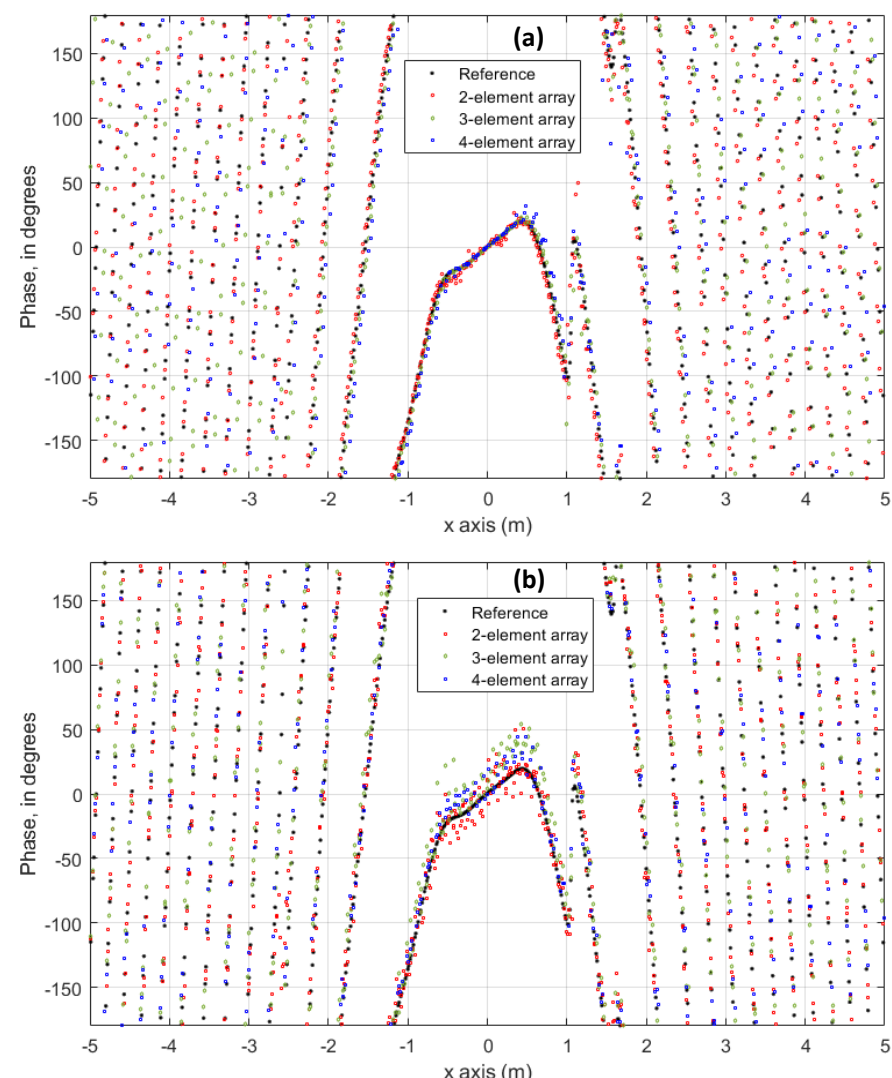

Fig. 5. Recovered phase. (a) No positioning errors. (b) Positioning errors assuming $\sigma_{\mathrm{pos}}=0.03 \lambda=5 \mathrm{~mm}$.

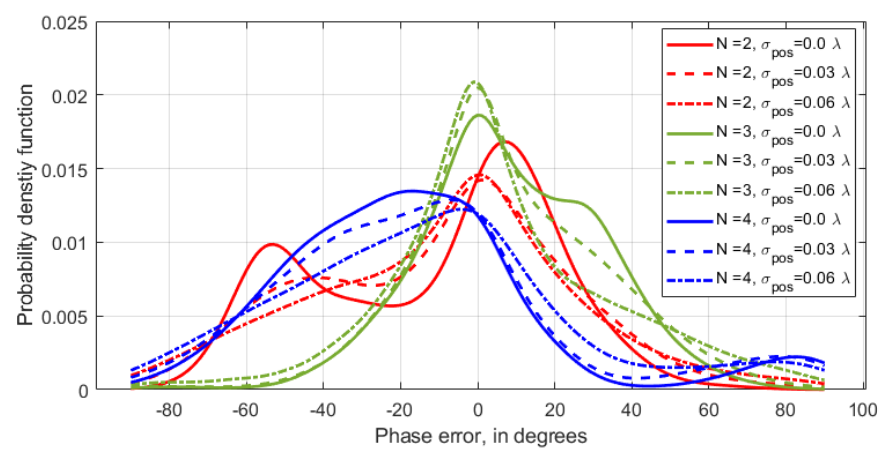

Fig. 6. Distribution of the phase error $\varepsilon_{\varphi}$ for different number of probe array elements, $N$, and different positioning errors, $\sigma_{\text {pos. }}$ Average phase error for 100 realizations of noise and positioning errors.

The average distribution of the phase error for 100 realizations of noise and positioning errors is shown in Fig. 6 for different number of array elements. It is observed that the dispersion of the phase error tends to increase with $\mathrm{N}$, especially between $N=3$ and $N=4$. This is due to the presence of noise in the acquired field samples, which has an impact in steps (2), (3), and (4). It has been verified that for noiseless NF acquisition considering $\Delta y=d$ the phase error is zero.

Next, from the recovered NF phase and acquired NF amplitude, the FF pattern is calculated. As shown in Fig. 7 (a), the error between the reference pattern and the calculated one is greater as $N$ increases. For $N=2$ and $N=3$, differences with respect to the reference pattern are less than $2 \mathrm{~dB}$ within the $\pm 30^{\circ}$ angular margin. The degree of accuracy in the reconstructed FF pattern is in the same order of magnitude as the one achieved with iterative phaseless techniques for this AUT (Fig. 5 of [13]).
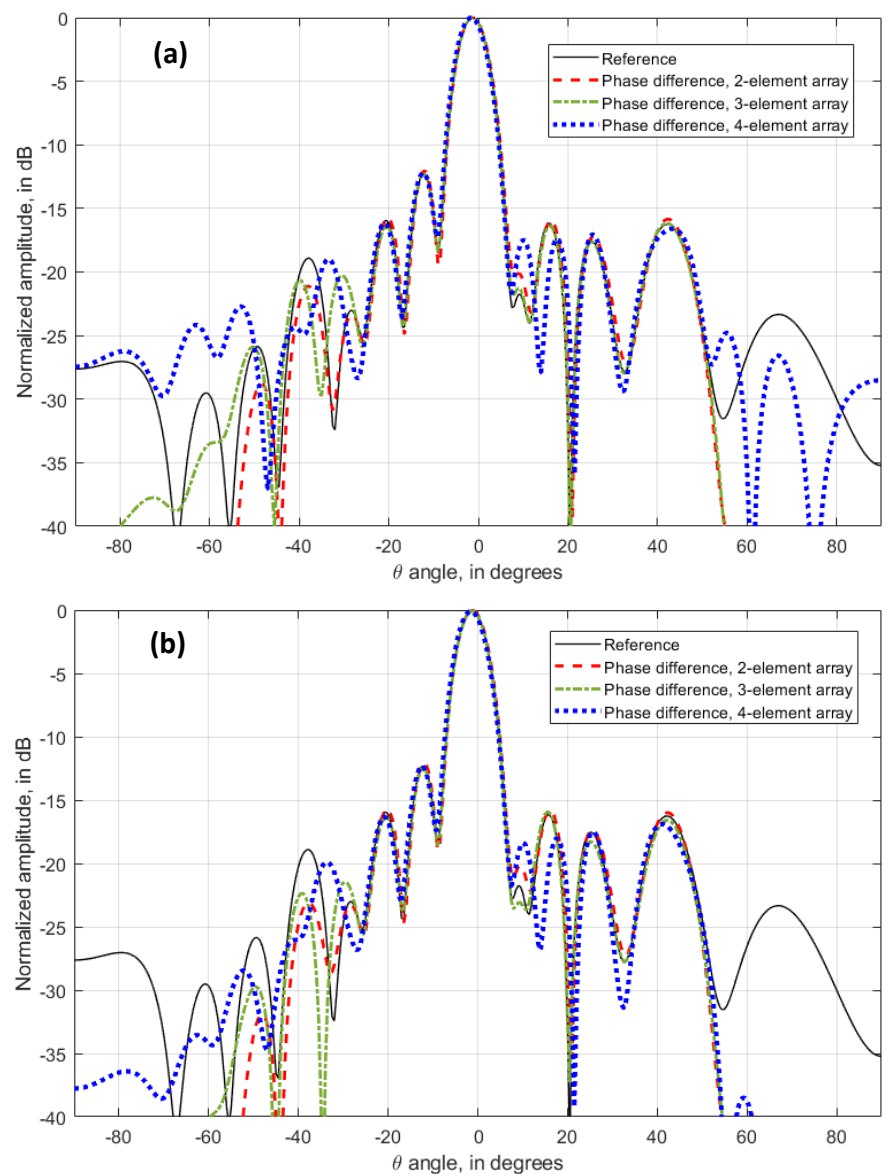

Fig. 7. AUT FF pattern calculated from acquired NF amplitude and recovered phase from acquired phase difference. (a) No positioning errors. (b) Positioning errors assuming $\sigma_{\mathrm{pos}}=0.06 \lambda=10 \mathrm{~mm}$. Average pattern for 100 realizations of noise and positioning errors.

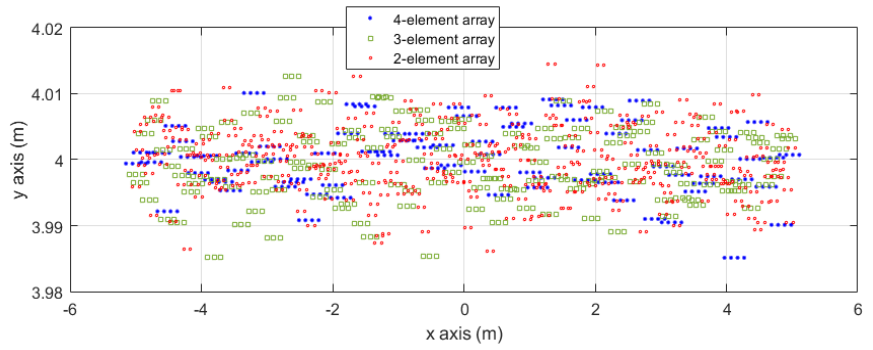

Fig. 8. Simulated probe array positioning errors, for $\sigma_{\mathrm{pos}}=0.03 \lambda=5 \mathrm{~mm}$.

As explained in Section I, the goal of the system proposed in this contribution is to enable in-situ complex antenna 
measurements using portable systems [3],[5]. That is why positioning uncertainty analysis, inherent to these portable systems, must be conducted to assess the impact in the accuracy of the phase retrieval technique. A Normal distribution of the positioning error with mean zero and standard deviation $\sigma_{\mathrm{pos}}$, has been considered (being $\sigma_{\mathrm{pos}}$ the same in $x$ and $y$ axes). Simulated positions, considering $\sigma_{\text {pos }}=$ $0.06 \lambda=5 \mathrm{~mm}$, are depicted in Fig. 8. As observed in Fig. 5 (b), positioning errors have an impact in the recovered phase, as can be inferred from the phase error distribution depicted in Fig. 6. The radiation pattern averaged for 100 realizations of positioning errors $\left(\sigma_{\mathrm{pos}}=0.06 \lambda\right)$ and different values of $\mathrm{N}$ is depicted in Fig. 7 (b).

\section{B. Two-dimensional case}

This subsection analyzes the extension of the methodology described in Section II from 1D to 2D measurements. An outline of the extension of the measurement system from 1D to 2D is depicted in Fig. 9. In this case, a $N \mathrm{x} N$ elements probe antenna array is required as phase differences must be measured along two orthogonal directions (e.g. along $x$ and $y$ axes). If the spatial variation of the amplitude of the measured field $(|\mathrm{E}|)$ is assumed to be slow, it can be measured at one of the $N \times N$ elements of the probe antenna array, as shown in Fig. 9. For each $m$-th measurement position, the phase at each element $\left(\varphi_{\mathrm{Pr}}\right)$ can be estimated from the phase difference between two adjacent elements, $\Delta \Phi_{\mathrm{Pr}}$, as indicated in Fig. 9 for a $2 \times 2$ probe antenna array. $\varphi_{\operatorname{Pr} 11 \text {,ref }}$ is the reference phase at each measurement position, which is set to $0^{\circ}$.

Phase retrieval from phase difference measurements is done as described in Section II.B, but in two steps: phase stitching is conducted first along one direction (e.g. $x$ axis), and then along the remaining direction (e.g. $y$ axis).

For the $2 \mathrm{D}$ analysis, a horn antenna working in the 1.7 to $2.5 \mathrm{GHz}$ frequency band has been selected as AUT (specifications of this AUT can be found in Section III.B of [18]). This antenna has been measured at spherical range in anechoic chamber at the frequency of $2.5 \mathrm{GHz}$, then reconstructing the fields on the AUT aperture [7]. From the aperture fields, the electric field has been calculated in a planar domain of $200 \times 240 \mathrm{~cm}$ placed $100 \mathrm{~cm}$ away from the aperture plane (see Fig. 9). As in the example of Section III.A, a SNR of $40 \mathrm{~dB}$ is considered.

A probe consisting of a $2 \times 2$ antenna array has been considered. As observed in the scheme depicted in Fig. 9, this configuration requires just one additional antenna, one power splitter and one phase difference detector with respect to the setup shown in Fig. 1 for linear (1D) acquisition. Spacing between antenna array elements is $d=5 \mathrm{~cm}(0.42 \lambda)$ in both $x$ and $y$ axes, to ensure proper sampling of the NF phase. Spacing between consecutive measurement positions is $\Delta x=$ $\Delta y=4 \mathrm{~cm}(0.33 \lambda)$, to ensure overlap. Positioning errors of $\sigma_{\text {pos }}=0 \lambda, 0.03 \lambda$, and $0.06 \lambda$ have been considered.

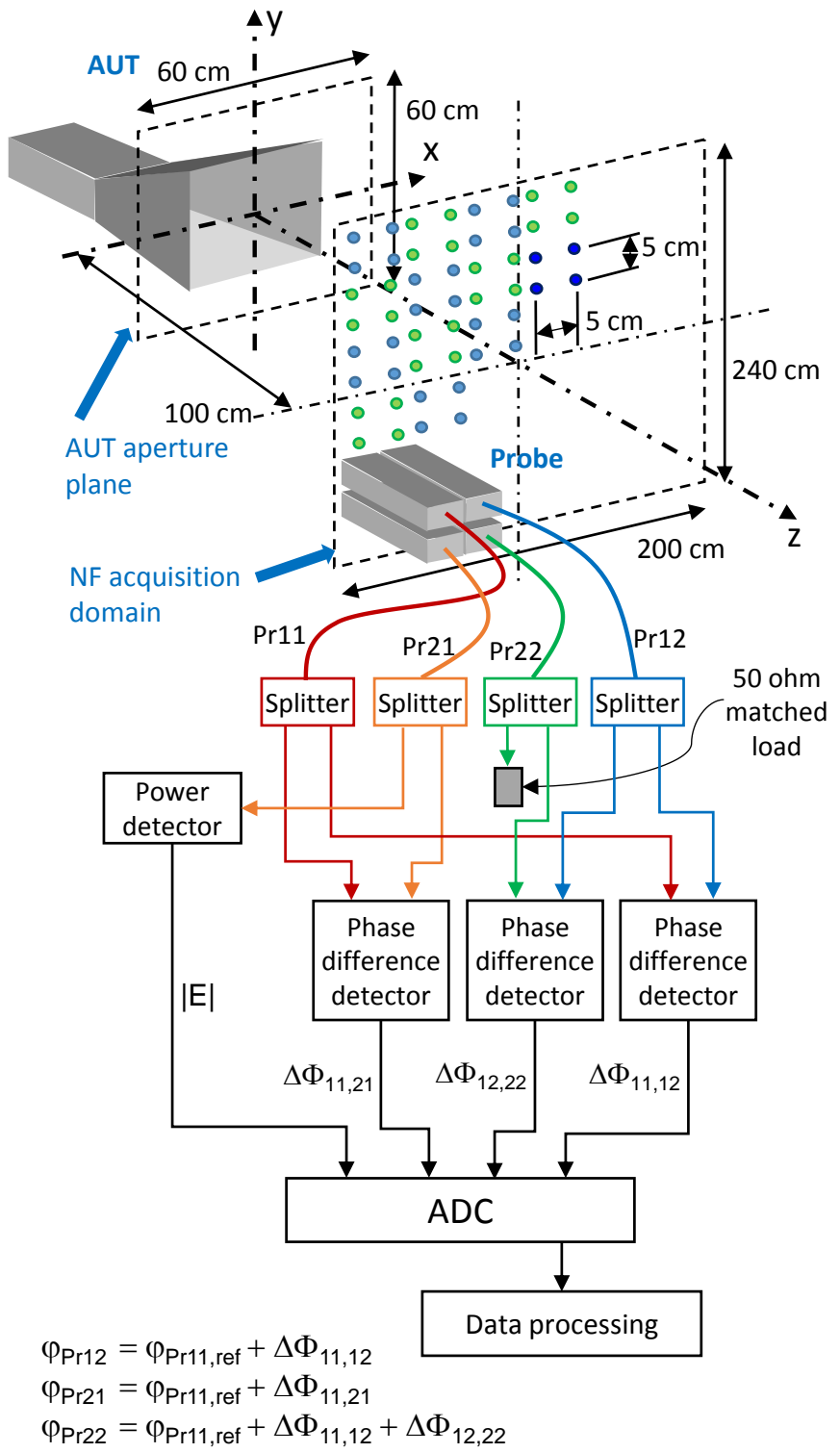

Fig. 9. Architecture of the proposed system for phase acquisition based on phase difference measurement, extended to $2 \mathrm{D}$ measurements. A probe antenna consisting of a $2 \times 2$ array is considered. A horn antenna has been chosen as AUT.

An example of the NF acquisition positions considering $\sigma_{\text {pos }}$ $=0.06 \lambda$ is shown in Fig. 10 (a), whereas the distribution of the phase error is plotted in Fig. 10 (b). The same behavior of the phase error as in Fig. 6 is observed. Besides, the phase of the $\mathrm{NF}$ at the acquisition positions (Fig. 10 (c)) is compared to the phase recovered using the proposed methodology (Fig. 10 (d)) when a positioning error of $\sigma_{\text {pos }}=0.06 \lambda$ is considered. 

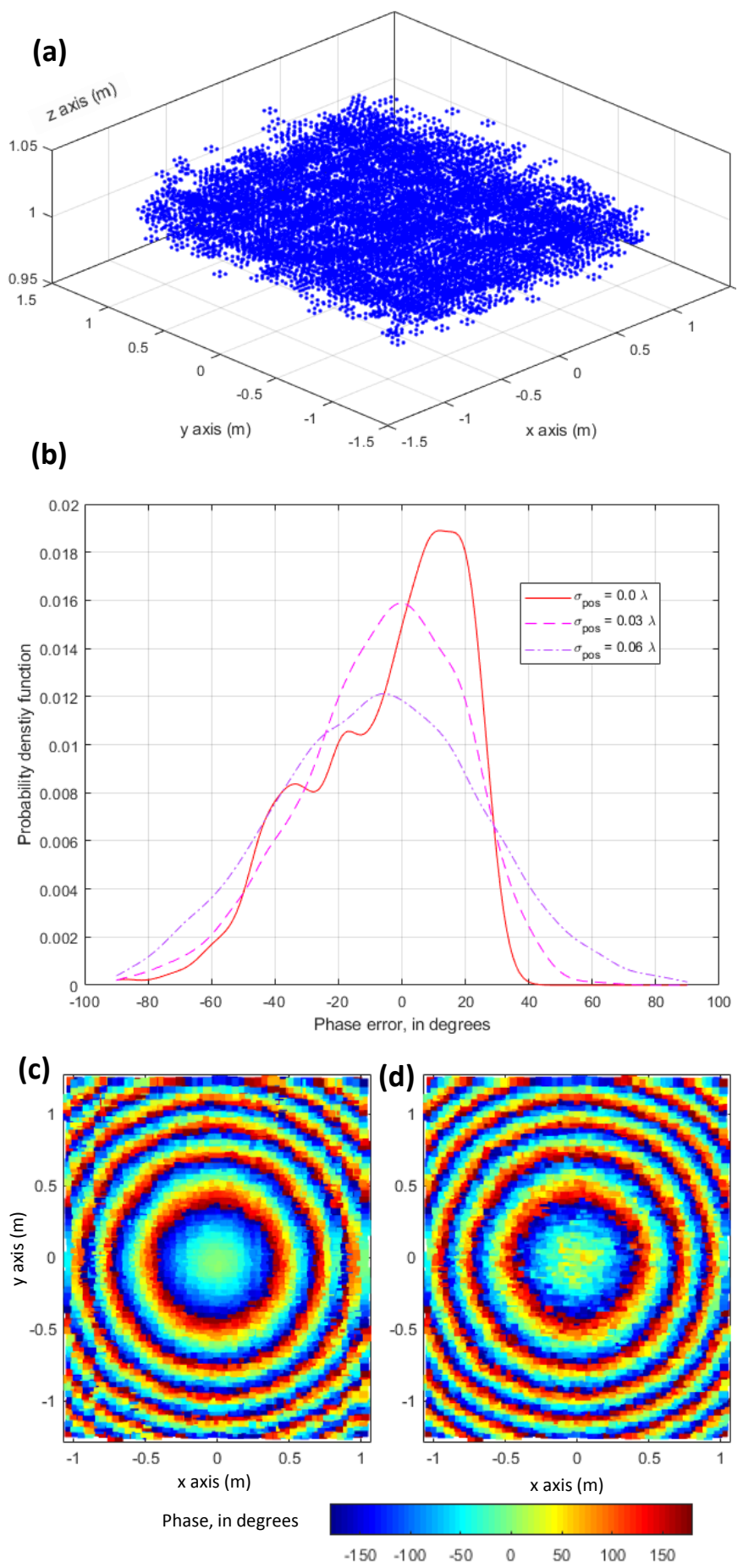

Fig. 10. (a) Placement of NF acquisition positions when a positioning error $\sigma_{\text {pos }}=0.06 \lambda=7 \mathrm{~mm}$ is considered. (b) Distribution of the phase error $\varepsilon_{\varphi}$ for different positioning errors, $\sigma_{\text {pos. }}$ (c) Phase of the acquired NF at each measurement position. (d) Reconstructed phase at each measurement position.
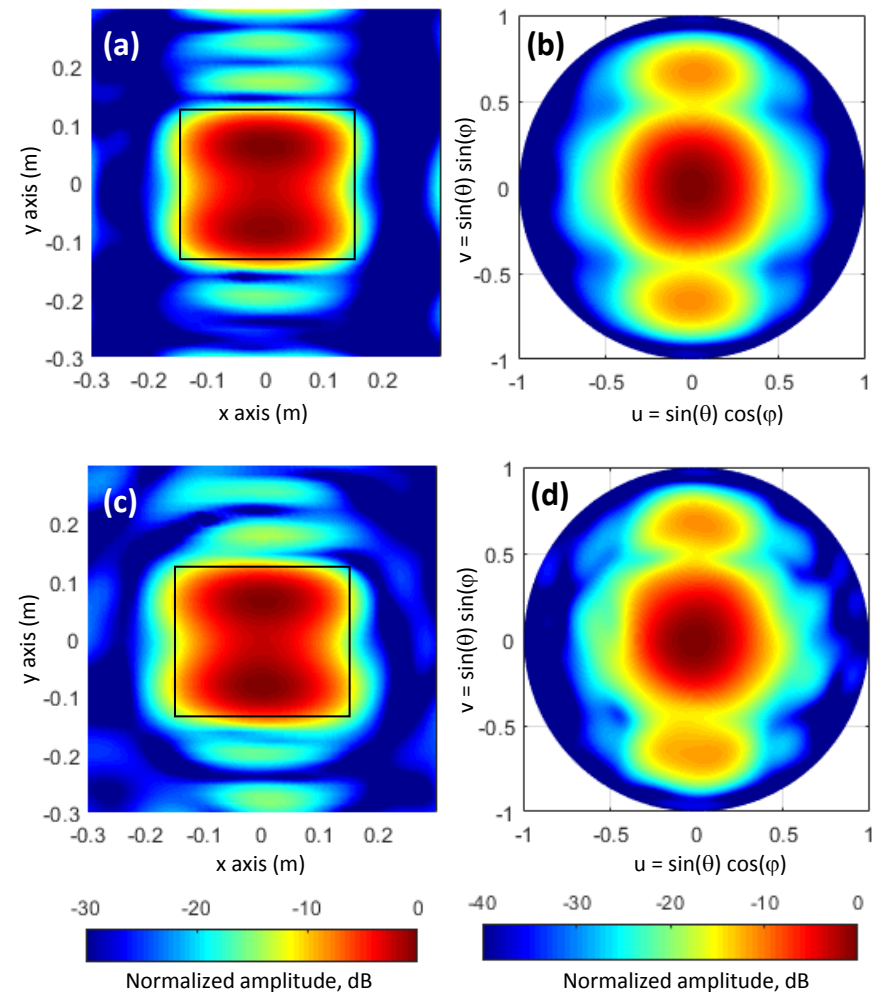

Fig. 11. Reconstructed fields on the aperture plane of the AUT from the recovered amplitude and phase using phase difference measurements: (a) no positioning error, (b) positioning error $\sigma_{\mathrm{pos}}=0.06 \lambda$. Black line represents the physical size of the horn antenna aperture. Far field pattern calculated from aperture fields: (b) no positioning error, (d) positioning error $\sigma_{\mathrm{pos}}=0.06 \lambda$.

Next, from the reconstructed NF, the fields on the AUT aperture plane have been reconstructed. Results are shown in Fig. 11 (a) and Fig. 11 (c) for the cases with no positioning error and with $\sigma_{\text {pos }}=0.06 \lambda$, respectively. From the aperture fields, the AUT radiation pattern is calculated (Fig. 12), comparing it with the reference pattern calculated from NF measurement at spherical range in anechoic chamber. Differences in the main lobe and secondary lobes between the reference pattern and the radiation pattern calculated from the recovered amplitude and phase using the proposed methodology are not greater than $1 \mathrm{~dB}$. In addition, the far field pattern calculated from iterative phase retrieval techniques using the amplitude of the NF measured at two different measurement distances [13] is plotted (Fig. 12, dashed black line) for comparison purposes.

The impact of positioning errors can be noticed also in the $2 \mathrm{D}$ representation of the radiation pattern: the $2 \mathrm{D}$ pattern with no positioning errors is depicted in Fig. 11 (b), and in Fig. 11 (d), the $2 \mathrm{D}$ pattern when $\sigma_{\mathrm{pos}}=0.06 \lambda$. 


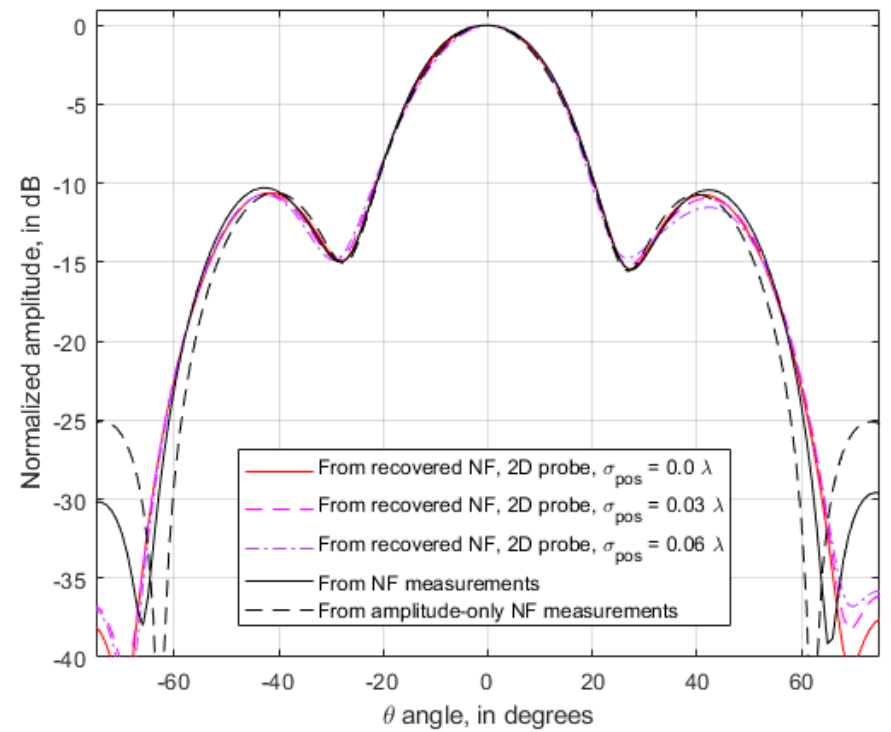

Fig. 12. Far field pattern calculated from aperture fields, E-plane $\left(\varphi=90^{\circ}\right)$.

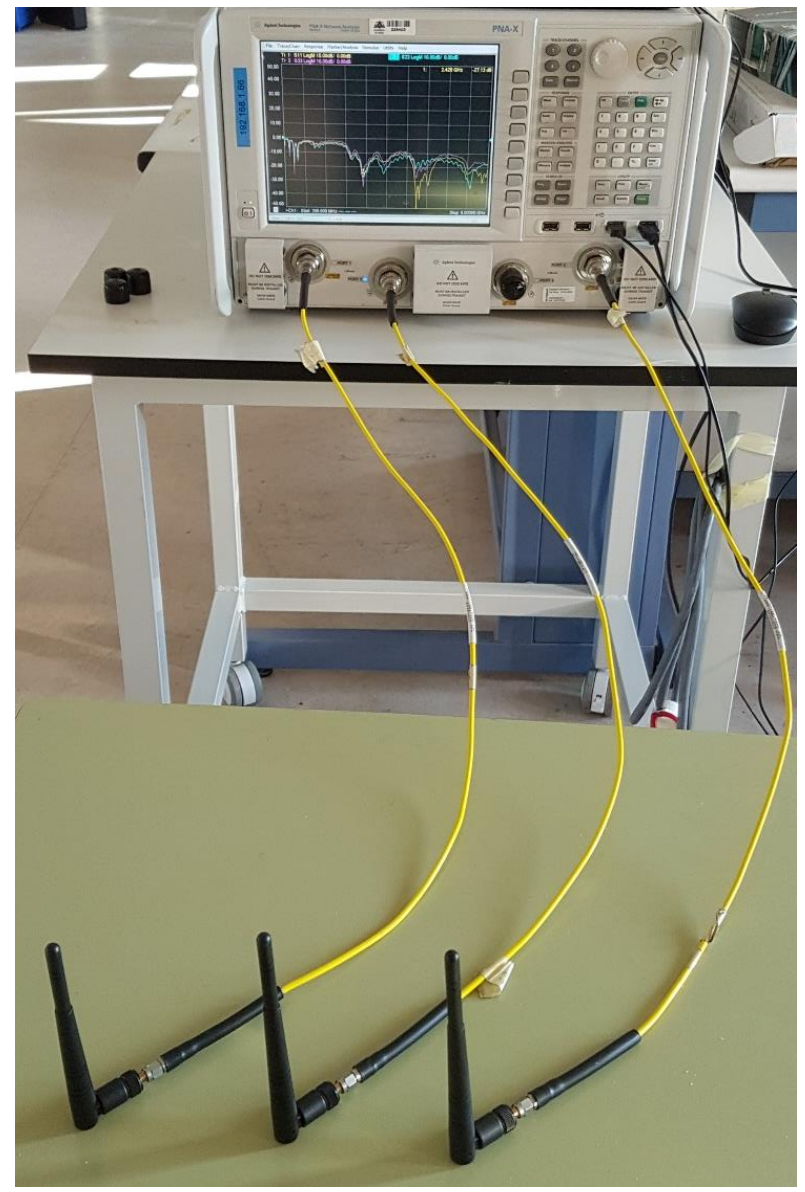

Fig. 13. Measurement of the $S_{11}$ parameter of the monopole antennas.

\section{IMPLEMENTATION AND EXPERIMENTAL VALIDATION}

\section{A. Hardware description}

The architecture depicted in Fig. 1 has been implemented using off-the shelf components. A value of $N=3$ has been chosen for the first implementation of the measurement system based on the results presented in Section III.A, and in order to compensate drift and uncertainties of phase difference measurements provided by each of the phase difference detectors. This results in a receiving unit composed by a threeelement antenna array, two phase difference detectors and a power detector.

A picture of the implemented receiver is shown in Fig. 14: the antenna array consists of three equally-spaced $(d=2.5 \mathrm{~cm})$ omni-directional antennas (in particular, commercial monopole antennas that can be used in WiFi equipment). The reasons why monopoles were chosen are: i) availability, as they are widely used for wireless applications at Industrial, Scientific and Medical (ISM) frequency bands, ii) trade-off between cost and performance (omni-directional radiation pattern, polarization purity).

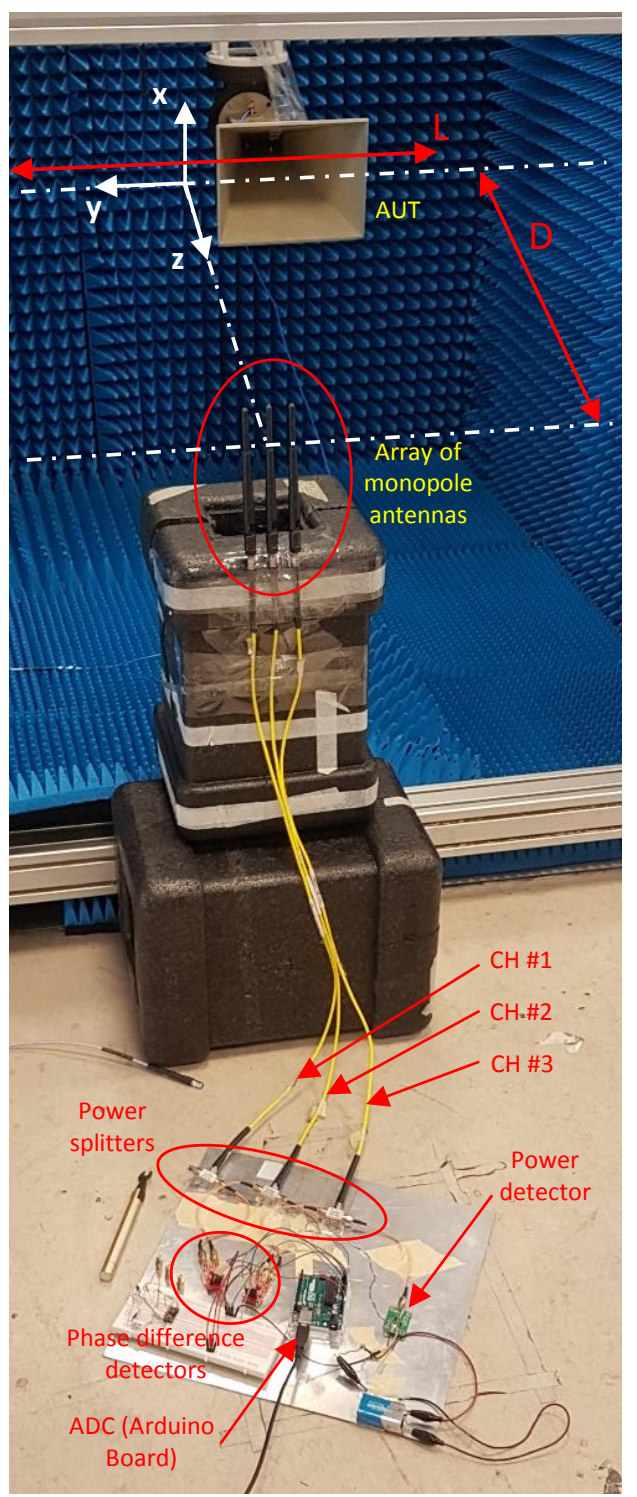

Fig. 14. Picture of the implemented system using off-the-shelf components, placed in a controlled environment for antenna measurement (XYZ measurement system).

The three monopole antennas are connected to three power splitters, whose output is connected to phase difference detectors [19] that provide a voltage proportional to the phase difference of the incident field in these antennas. One power 
splitter is also connected to a power detector [20] that provides a voltage related to the field intensity. Aiming to keep costeffective requirements, the phase difference detectors selected for this setup can work up to $2.7 \mathrm{GHz}$. The selected commercial monopole antennas are matched at $2.4-2.5 \mathrm{GHz}$ frequency band, so that the selected working frequency for validation purposes is $2.45 \mathrm{GHz}$ (thus $d=0.2 \lambda$ ). Measurement of the $S_{11}$ parameter at the output of each monopole and RF cable has been measured using a 4-port Vector Network Analyzer (VNA) to check that their amplitude and phase responses in the frequency band of interest are the same (Fig. 13). The $S_{21}$ parameter of each coaxial cable has been measured to verify that the frequency response of these cables is the same.

Analog-to-Digital conversion (ADC) is based on an Arduino board, keeping the cost-effective requirement of the implemented receiver (less than 200 EUR).
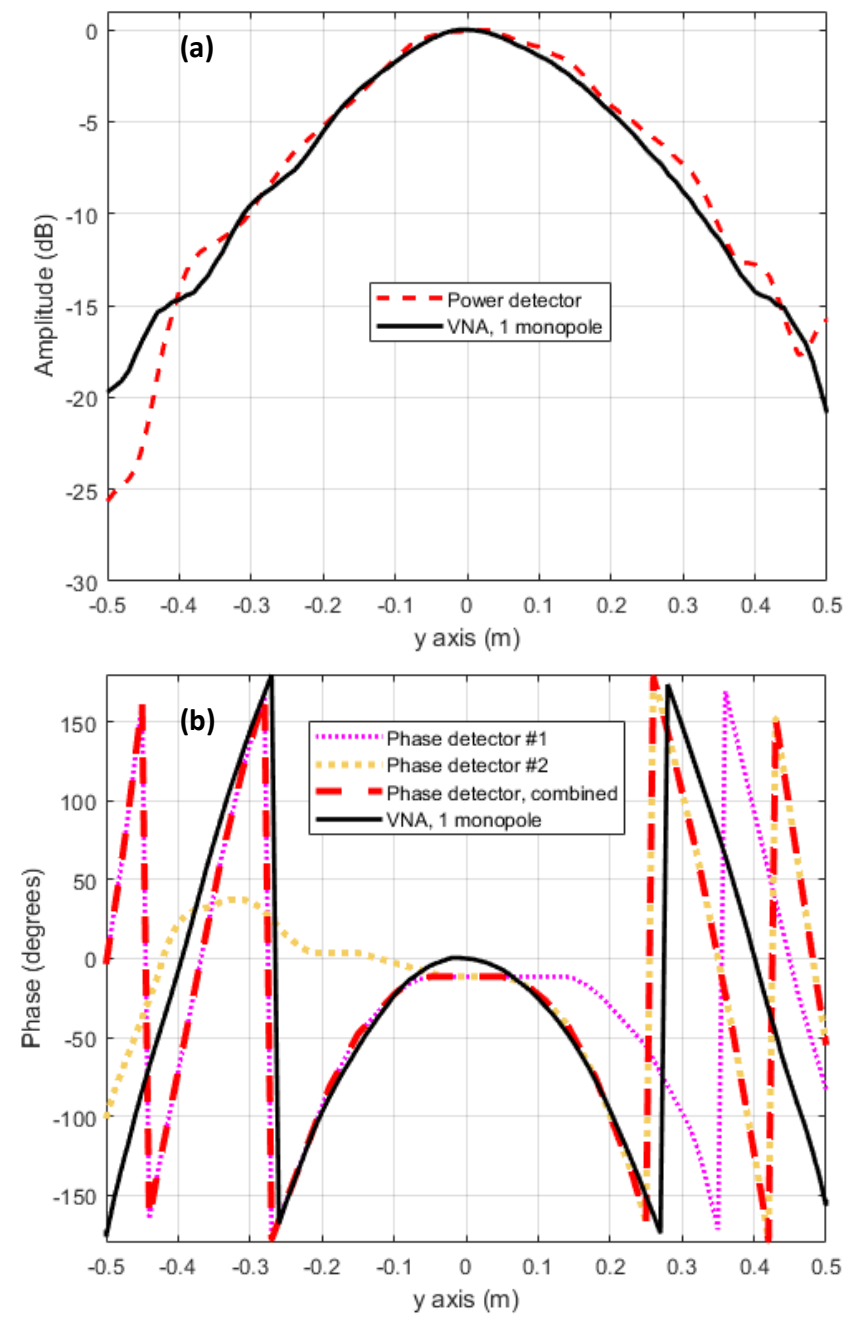

Fig. 15. Measured NF amplitude and phase of the AUT over a rectilinear path, $D=50 \mathrm{~cm}$. Comparison between measurements using the implemented receiver and measurements with a VNA and a single monopole as probe antenna.

\section{B. Evaluation of the implemented receiver}

In order to assess the accuracy of the implemented receiver, measurements in a controlled environment have been conducted (Fig. 14). A pyramidal horn antenna working in the 2-4 GHz frequency band has been selected as AUT. For the working frequency of $2.45 \mathrm{GHz}$ and given the AUT size, FF distance is around $1.5 \mathrm{~m}$. Taking advantage of the positioning capabilities of the XYZ measurement system [21], the field radiated by the AUT has been assessed at two different distances, $D=50 \mathrm{~cm}$ and $D=70 \mathrm{~cm}$, along a straight line of $L$ $=1 \mathrm{~m}$ in the $y$-axis, sampled every $1 \mathrm{~cm}$, which satisfies (1). This measurement corresponds to the acquisition of the copolar component of the AUT in the H-plane.

The XYZ measurement system positioning uncertainty is less than $10 \mu \mathrm{m}$, so for the working frequency of $2.45 \mathrm{GHz}$, positioning errors are negligible. Measurement positions are given by the XYZ measurement system positioning controller unit.

The NF radiated by the AUT measured at $D=50 \mathrm{~cm}$ is represented in Fig. 15. Amplitude is directly given by the power detector, after converting the output voltage into $\mathrm{dBm}$, according to [20]. For comparison purposes, the same measurement has been conducted using a VNA and a single monopole as a probe antenna (the other two monopoles were removed). The agreement between the measured amplitude using the power detector (Fig. 14) and the VNA can be observed in Fig. 15 (a), despite no probe correction has been applied.

Phase measurements are plotted in Fig. 15 (b). For each phase difference detector, the output voltage is converted into a phase difference value [19], then applying the methodology described in Section II.B. With the proposed receiver architecture, an asymmetry in the recovered phase is noticed, which can be due to the mutual coupling effects in the antenna array. The recovered phase is compared with VNA measurements in Fig. 15 (b), observing that part of the recovered phase on each phase difference detector agrees the one measured with the VNA.

\section{Modification of the proposed architecture}

Aiming to avoid the asymmetry observed with the setup implemented in Fig. 14, another architecture for the receiver has been tested (Fig. 16). The 3-element antenna array is kept, but now, the center monopole is directly connected to the power detector, and the outer monopoles are connected to a phase difference detector $(N=2,2 d=5 \mathrm{~cm})$. This configuration tries to maximize the symmetry between phase difference detector inputs and, in addition, it also simplifies the hardware of the receiver. A picture of the modified receiver is shown in Fig. 17. For comparison purposes, a power splitter has been placed to conduct amplitude measurements with the VNA and the power detector simultaneously. In this case, VNA measurements have been conducted using first the 3-element array as probe and then using a single monopole as probe, aiming to assess precisely the impact of probe pattern effect in the measurements. 


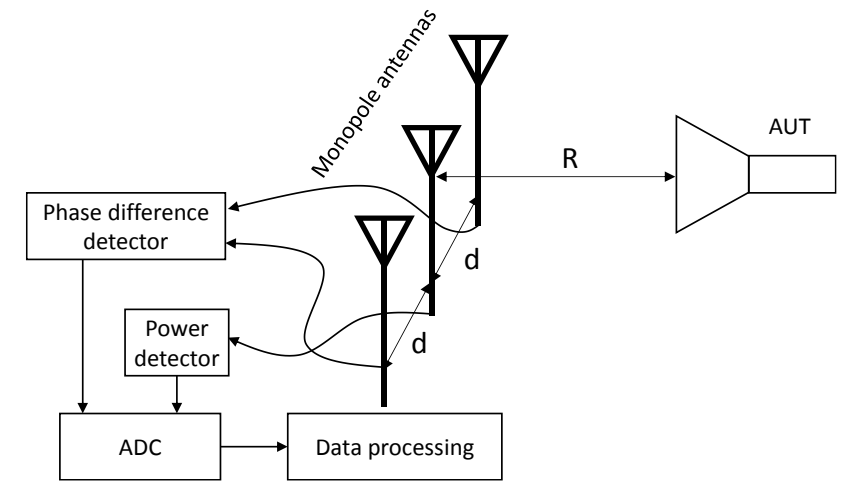

Fig. 16. Architecture of the modified receiver.
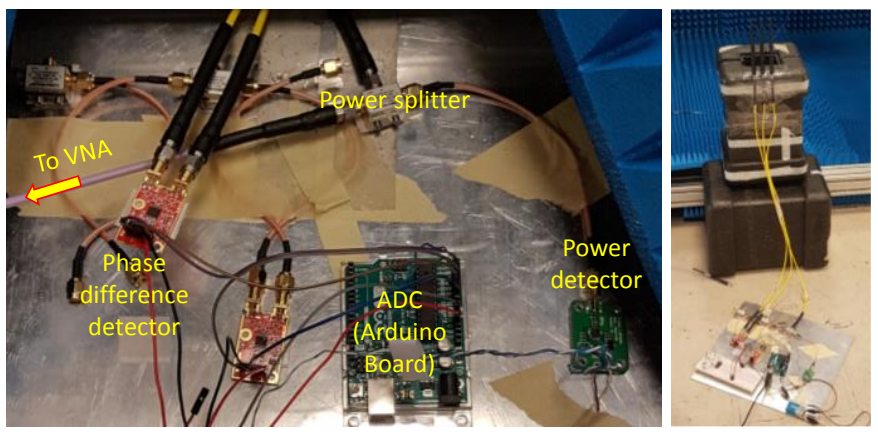

Fig. 17. Picture of the modified receiver.

Measurement results are depicted in Fig. 18. It can be observed that the probe pattern affects the measured amplitude, regardless the kind of sensor connected at the output of the probe antenna (Fig. 18 (a), dashed lines). However, probe pattern has not an impact in phase measurements, as noticed in Fig. 18 (b). This result is of special interest for the proposed receiver, as correction of the potential phase distortion introduced by the probe pattern is more complex than amplitude correction.

The amplitude of the measured field $\left(E_{\text {meas }}(\boldsymbol{r})\right)$ can be corrected $\left(E_{\text {corr }}(\boldsymbol{r})\right)$ [22] if the probe pattern is known $\left(E_{\text {probe }}(\boldsymbol{r})\right)$, as indicated in (6):

$$
\left|E_{\text {corr }}(r)\right|=\left|E_{\text {meas }}(r)\right| /\left|E_{\text {probe }}(r)\right|
$$

For this 3-element monopole antenna array, $E_{\text {probe }}(\boldsymbol{r})$ has been calculated using a Method-of-Moments software [22]. Fig. 19 represents the field radiated by the 3 monopoles (taking into account mutual coupling), which is introduced in (6) to correct the probe pattern distortion. Corrected amplitude is depicted in Fig. 18 (a), where the agreement with the amplitude measured with a single monopole can be noticed.

Phase measurements using the VNA with a single monopole and the 3-element probe, as well as using the modified receiver architecture, are plotted in Fig. 18 (b). Phase error is less than $15^{\circ}$ within $y= \pm 30 \mathrm{~cm}$. Measurement results at $D=70 \mathrm{~cm}$ are plotted in Fig. 20. In this case, the phase error between VNA measurements and the phase retrieved using the modified setup is greater (up to $30^{\circ}$ ) within the interval $y= \pm 30 \mathrm{~cm}$.
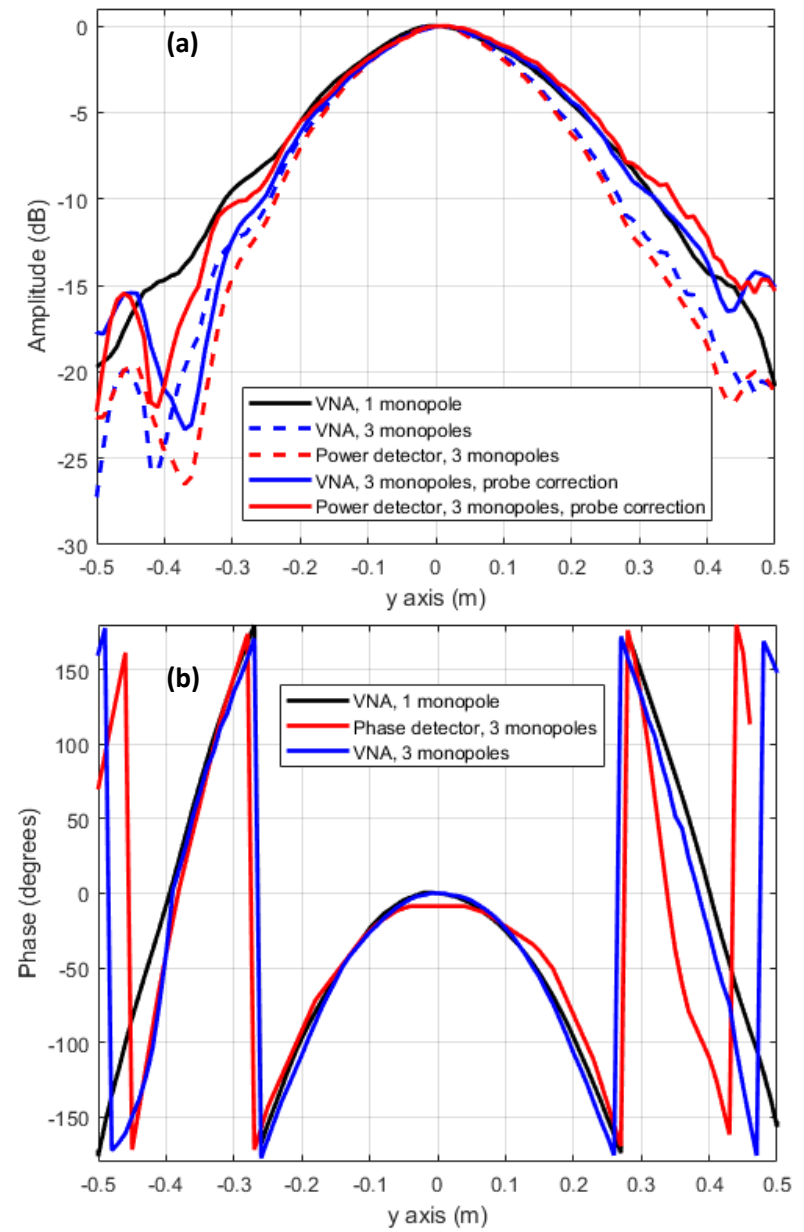

Fig. 18. Measured NF amplitude and phase of the AUT, $D=50 \mathrm{~cm}$. Comparison between measurements using the implemented receiver and using a VNA, considering both a single monopole antenna and a 3-element monopole array as probe antenna.
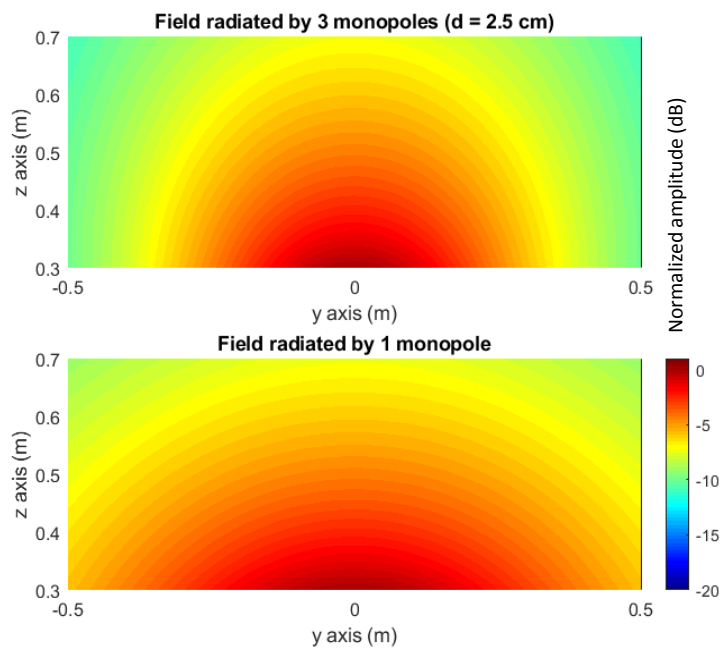

Fig. 19. Field radiated by the probe antenna in the AUT measurement domain. Comparison with the field radiated by a single (omni-directional) monopole antenna.

\section{AUT diagnostics and radiation pattern calculation}

As explained in Section I, NF measurements are postprocessed to obtain antenna diagnostics information and/or the 
radiation pattern of the AUT. In this subsection, the NF measured with the VNA and with the implemented receiver will be used to recover the field on the aperture plane $(z=0 \mathrm{~m})$ of the AUT $\left(E_{\text {ap }}\right)$ by means of NF-NF transformation, and the FF pattern (H-plane cut) by means of NF-FF transformation.

Complex NF measurements (that is, when amplitude and phase are known) can be post-processed using the Sources Reconstruction Method (SRM) [7]. In order to maximize the angular margin of validity $\left(\theta_{\mathrm{FF}}\right)$, measurements (both conducted with the VNA and with the implemented receiver), taken at $D=50 \mathrm{~cm}$ have been considered (yielding $\theta_{\mathrm{FF}}= \pm 45^{\circ}$ ).

In addition, and for comparison purposes, an iterative phase retrieval technique, the phaseless SRM [13], has been tested taking the amplitude of the NF measured at $D=50 \mathrm{~cm}$ and $D$ $=70 \mathrm{~cm}$ (solid red line, Fig. 18 (a) and Fig. 20 (a)).

Aperture fields (co-polar component, H-plane) are depicted in Fig. 21 (a), where it is observed that the agreement between aperture fields from VNA measurements (black line) and using the implemented receiver (red line) is better than the aperture fields recovered from amplitude-only measurements (green line). Concerning FF pattern (co-polar component, H-plane), Fig. 21 (b), the same behavior is noticed.
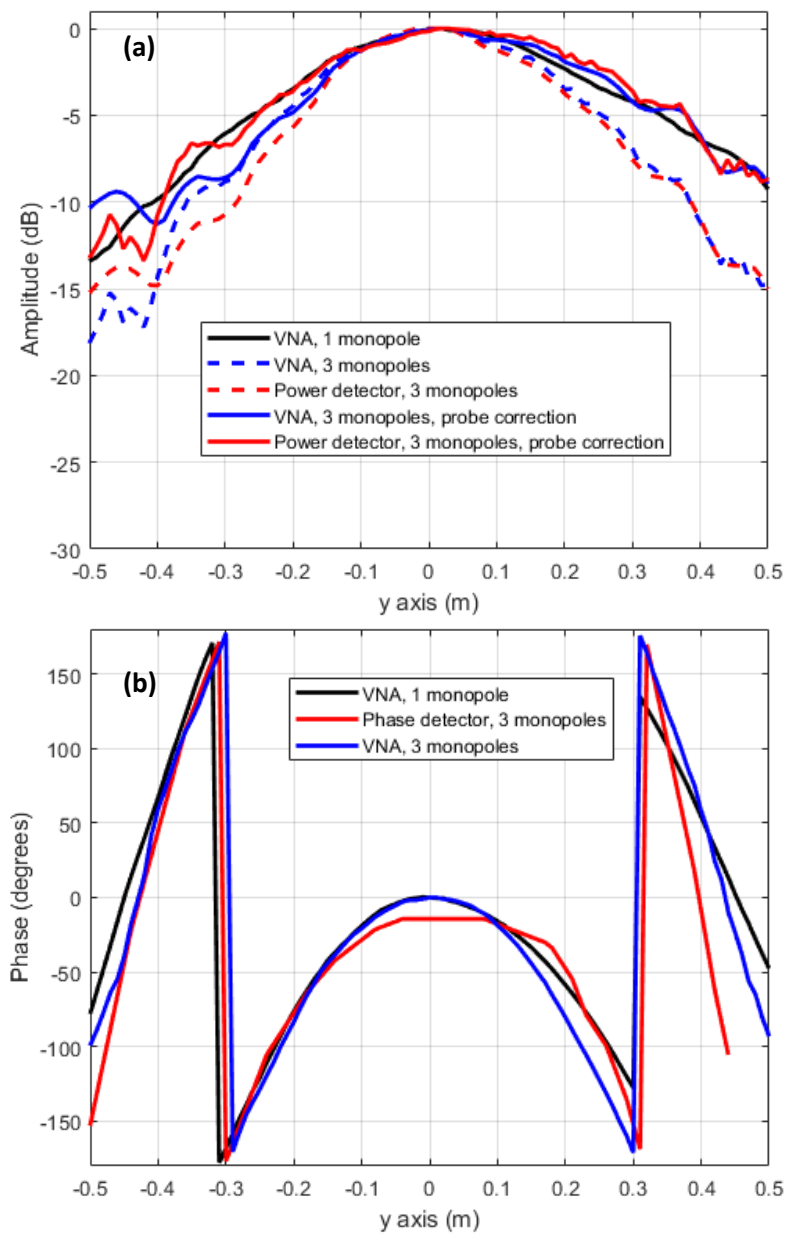

Fig. 20. Measured NF amplitude and phase of the AUT, $D=70 \mathrm{~cm}$. Comparison between measurements using the implemented receiver and measurements with a VNA considering a single monopole antenna probe, and considering the 3 -element monopole antenna array.

\section{E. Antenna array measurement and characterization}

The presence of points where the amplitude of the NF is negligible (e.g. nulls) impacts the performance of the methodology presented in this contribution, as the phase reference is lost at these points. Nevertheless, it must be pointed out that NF measurements exhibit null filling, as the radiation pattern is not still formed. Nulls in the NF may still appear due to the excitation law of the elements of antenna arrays, or due to the geometry of the antenna.

In order to assess the performance of the phase difference detector when there is a $180^{\circ}$ degree phaseshift in the measured NF, a second AUT has been tested. The AUT is an array composed by 2 parallel wideband logarithmic periodic antennas as elements, working in the $1-6 \mathrm{GHz}$ frequency band. The spacing between elements is $10 \mathrm{~cm}$, larger than $\lambda / 2$ at $2450 \mathrm{MHz}$ to ensure the presence of grating lobes (and thus a null in the pattern).

NF measurements have been conducted at a distance of $D=$ $70 \mathrm{~cm}$, along a straight line of $L=1.2 \mathrm{~m}$ in the $y$-axis (see Fig. 22 ), so that $\theta_{\mathrm{FF}}= \pm 41^{\circ}$. Apart from the receiver implemented as described in Section IV.C, the AUT has been measured using the VNA, considering both a single monopole and a 3-element monopole antenna array as probe.
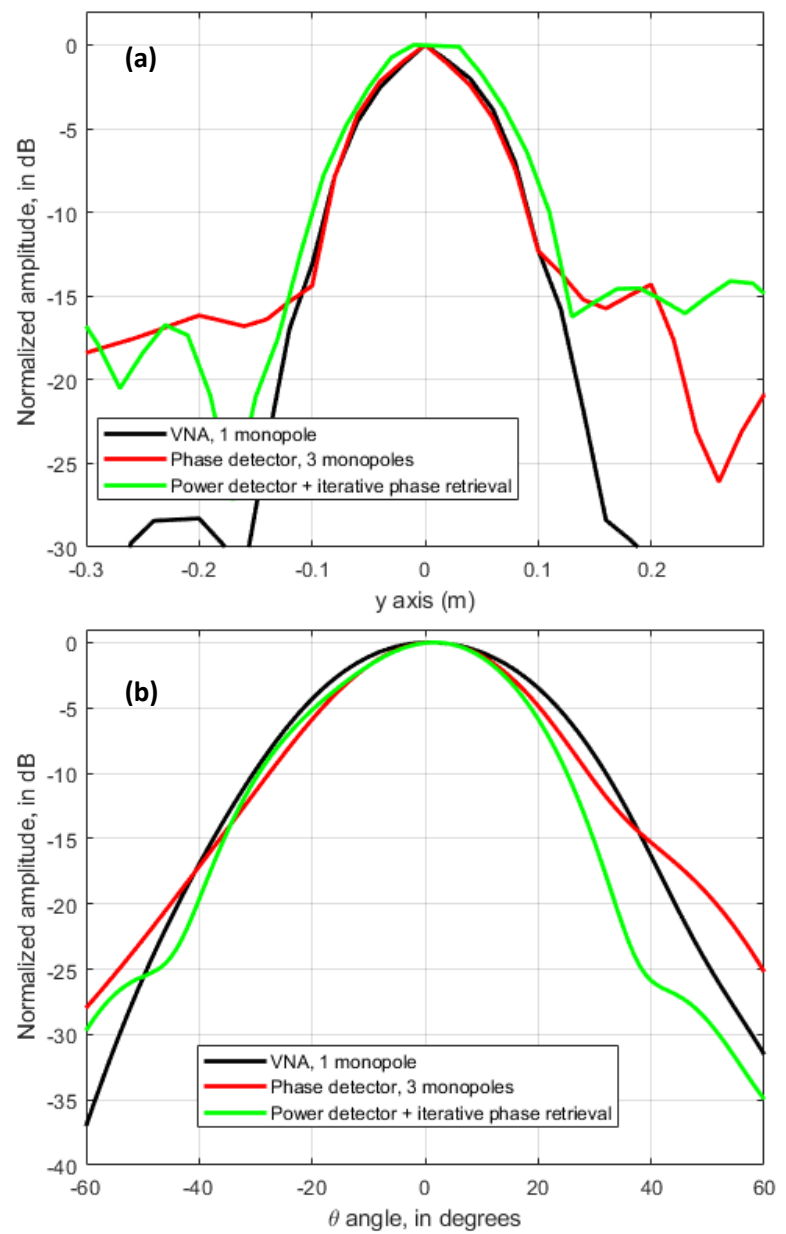

Fig. 21. (a) Reconstructed aperture fields. (b) Far field pattern, H-plane cut. 


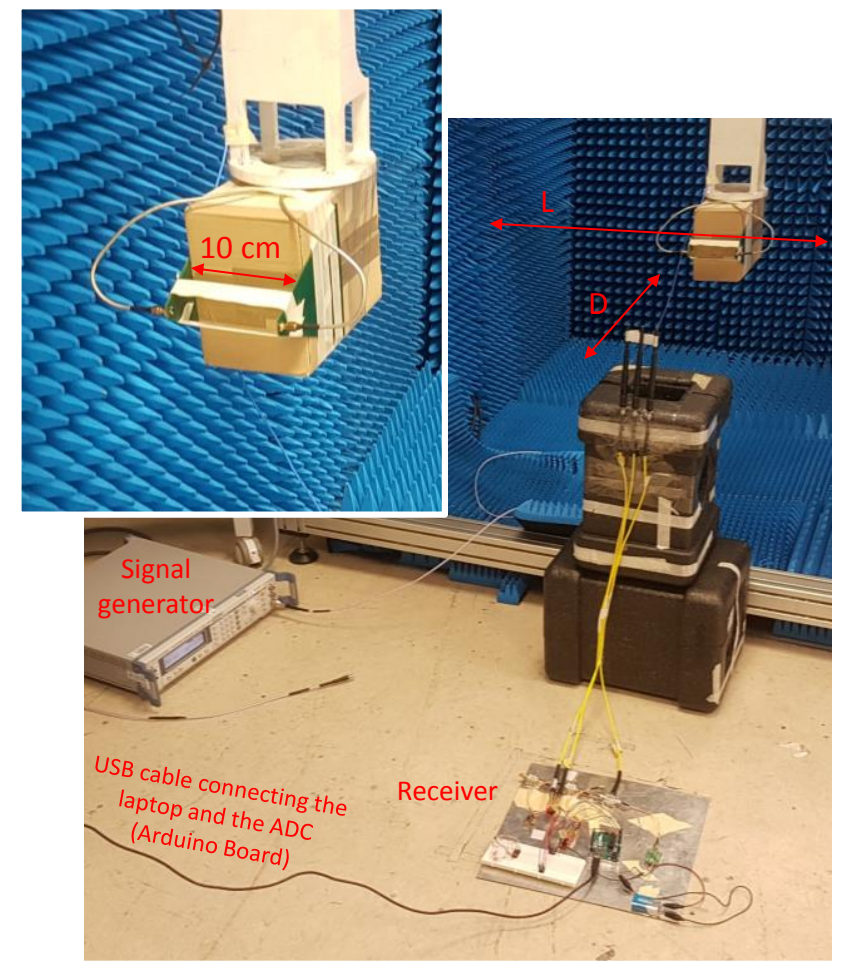

Fig. 22. Picture of the 2-element array chosen as AUT, and the measurement setup using the implemented receiver.

NF measurements are plotted in Fig. 23. Amplitude measurements using the VNA with a single monopole, and probe pattern corrected measurements taken with the 3monopole array probe (using both the VNA and the power detector) are depicted in Fig. 23 (a). Misbalance in the feeding network of the AUT resulted in a null appearing at around $y=$ $5 \mathrm{~cm}$. In the case of phase measurements, there is a $180^{\circ}$ shift in the null. As expected, the phase difference detector cannot track this sharp variation, so the $180^{\circ}$ shift is partially filled, as observed in Fig. 23 (b). Nevertheless, for post-processing purposes, this wrong phase is linked to very low amplitude values (the null of the measurements).
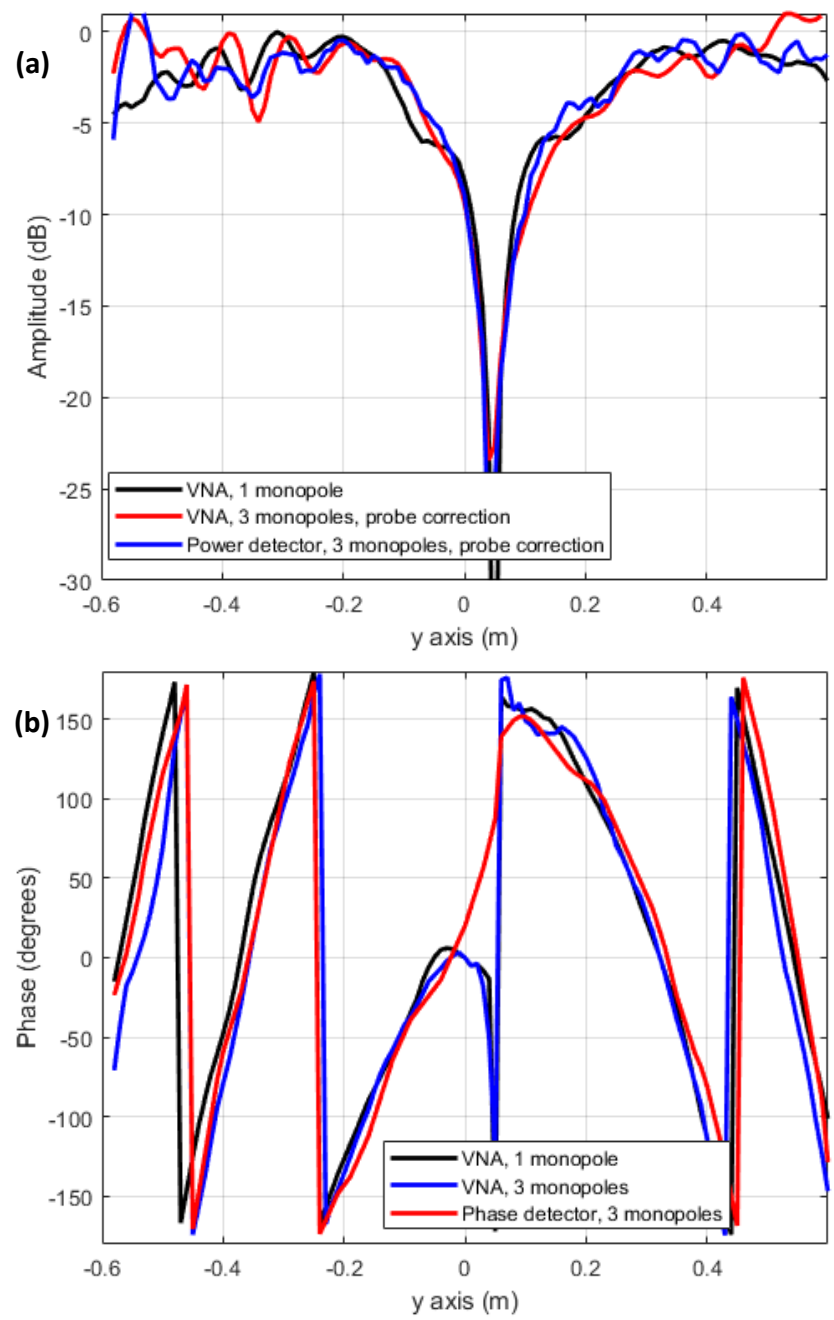

Fig. 23. Measured NF amplitude and phase of the AUT, $D=70 \mathrm{~cm}$. Comparison between measurements using the implemented receiver and measurements with a VNA considering a single monopole antenna probe, and considering the 3 -element monopole antenna array.

Finally, from the measured NF amplitude and the recovered phase, aperture fields and FF pattern are calculated, as done in Section IV.D. Reconstructed aperture fields are plotted in Fig. 24 (a), comparing results from VNA and implemented receiver measurements. The placement of each element of the AUT can be identified ( $y_{\text {element, } 1}=-1 \mathrm{~cm}, y_{\text {element, } 2}=9 \mathrm{~cm}$ ). In the case of the FF pattern, Fig. 24 (b), the location of the grating lobes can be observed, being the error in the calculated FF not greater than $5 \mathrm{~dB}$ in the angular margin of validity. 

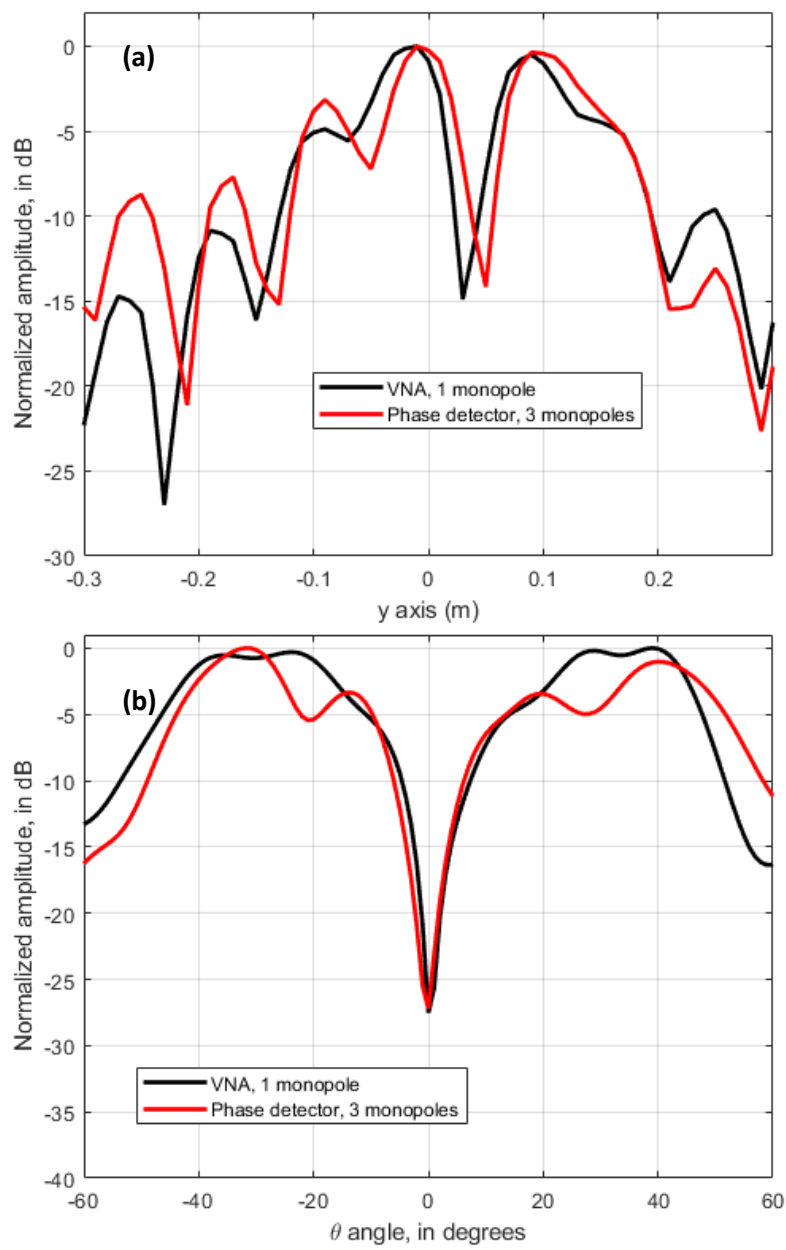

Fig. 24. (a) Reconstructed aperture fields. (b) Far field pattern, H-plane cut.

\section{CONCLUSIONS}

This contribution has proved the feasibility of complex field measurements with a cost-effective receiver architecture based on amplitude and phase difference detectors. The possibility of recovering phase information by combining phase difference measurements also reduces measurement time with respect to those systems based on iterative phase retrieval methods, as just one acquisition is needed.

Concerning experimental validation, several issues affecting the accuracy of the measurements were found after the implementation and testing of the proposed receiver with offthe-shelf components. These issues could be partially overcome by modifying the architecture of the receiver.

Despite not being as accurate as dedicated equipment for antenna measurement (e.g. a VNA), the proposed receiver can be used for preliminary assessment of the AUT. Furthermore, it must be remarked that no physical connection between the AUT and the implemented receiver is needed, so it can be integrated into portable platforms and devices for in-situ antenna measurement.

\section{REFERENCES}

[1] M. A. Salas-Natera, R. M. Rodriguez-Osorio, L. De Haro, "Procedure for Measurement, Characterization, and Calibration of Active Antenna
Arrays". IEEE Transactions on Instrumentation and Measurement, Vol. 62, Issue 2, pp. 377-391, 2013.

[2] J. Borowich, A. E. Holley, W. L. Lange, R. W. Howard, R. L. Cummings, "A Near-Field Antenna Measurement System," IEEE Transactions on Instrumentation and Measurement, Vol. 32, Issue 1, pp. 91-102, 1983.

[3] G. Álvarez-Narciandi, J. Laviada, Y. Alvarez-Lopez, F. Las-Heras, "Rapidly Deployable Portable System for Real-Time Antenna Diagnostics and Characterization," Proc. of the 2019 IEEE Antennas and Propagation and USNC-URSI Radio Science Meeting, Atlanta (USA), 7-12 July 2019, pp. 1-2.

[4] L. Ciorba, G. Virone, F. Paonessa, S. Matteoli, P. Bolli, et al., "NearField Phase Reconstruction for UAV-based Antenna Measurements", in Proc. Of the $13^{\text {th }}$ European Conference on Antennas and Propagation, Krakow, Poland, 31 March-5 April 2019. pp. 1-5.

[5] M. García, Y. Álvarez, F. Las-Heras, "On the use of Unmanned Aerial Vehicles for Antenna and Coverage Diagnostics in Mobile Networks," IEEE Communications Magazine, Vol. 56, No. 7, pp. 72-78, 2018.

[6] A. Jam, K. Sarabandi, "A submillimeter-wave near-field measurement setup for on-wafer pattern and gain characterization of antennas and arrays," IEEE Transactions on Instrumentation and Measurement, Vol. 66, Issue 4, pp. 802-811, 2017.

[7] Y. Alvarez, F. Las-Heras, M. R. Pino, T. K. Sarkar, "An Improved Super-Resolution Source Reconstruction Method," IEEE Transactions on Instrumentation and Measurement, Vol. 58, Issue 11, pp. 38553866, 2009.

[8] J. Laviada, F. Las-Heras, "Phaseless antenna measurement on nonredundant sample points via Leith-Upatnieks Holography," IEEE Transactions on Antennas and Propagation, Vol. 61, No. 8, pp. 40364044, 2013.

[9] D. Smith, M. Leach, M. Elsdon, S. J. Foti, "Indirect holographic techniques for determining antenna radiation characteristics and imaging aperture fields," IEEE Antennas and Propagation Magazine, Vol. 49, No. 1, pp. 54-67, 2007.

[10] J. Laviada Martínez, A. Arboleya-Arboleya, Y. Álvarez-López, C. García-González, and F. Las-Heras, "Phaseless antenna diagnostics based on off-axis holography with synthetic reference wave," IEEE Antennas and Wireless Propagation Letters, Vol. 13, pp. 43-46, 2014.

[11] A. Arboleya, J. Laviada, J. Ala-Laurinaho, Y. Álvarez, F. Las-Heras, Antti, V. Räisänen, "Phaseless characterization of broadband antennas". IEEE Transactions on Antennas and Propagation, Vol. 64, No. 2, pp. 484-495, 2016.

[12] S. F. Razavi, Y. Rahmat-Samii, "A new look at phaseless planar nearfield measurements: limitations, simulations, measurements, and a hybrid solution," IEEE Antennas and Propagation Magazine, Vol. 49, Issue 2, pp. 170-178, 2007.

[13] Y. Álvarez, F. Las-Heras, M. R. Pino, "The Sources Reconstruction Method for Amplitude-Only Field Measurements," IEEE Transactions on Antennas and Propagation. Vol. 58, No. 8, pp. 2776-2781, 2010.

[14] T. Brown, I. Jeffrey, P. Mojabi, "Multiplicatively regularized sources reconstruction method for phaseless planar near-field antenna measurements," IEEE Transactions on Antennas and Propagation, Vol. 65, No. 4, pp. 2020-2031, 2017.

[15] A. Capozzoli, C. Curcio, G. D’Elia, A. Liseno, "Millimeter-wave phaseless antenna characterization," IEEE Transactions on Instrumentation and Measurement, Vol. 57, Issue 7, pp. 1330-1337, 2008.

[16] S. Costanzo, G. Di Massa, "Wideband phase retrieval technique for amplitude-only near-field data," Radioengineering, Vol. 17, No. 4, pp. $8-12,2008$

[17] S. Costanzo, G. Di Massa, M. D. Migliore, "A novel hybrid approach for far-field characterization from near-field amplitude-only measurements on arbitrary scanning surfaces," IEEE Transactions on Antennas and Propagation, Vol. 53, No. 6, pp 1866-1874, 2005.

[18] F. Las-Heras, M. R. Pino, S. Loredo, Y. Alvarez, T. K. Sarkar, "Evaluating near-field radiation patterns of commercial antennas," IEEE Transactions on Antennas and Propagation, Vol. 54, No. 8, pp. 21982207, 2006.

[19] Analog Devices. AD8302. LF-2.7 GHz RF/IF Gain and Phase Detector. Datasheet available at: https://www.analog.com/media/en/technicaldocumentation/data-sheets/ad8302.pdf [Accessed 15-09-2019]. 
[20] Analog Devices. AD8318. $1 \mathrm{MHz}$ to $8 \mathrm{GHz} 70 \mathrm{~dB}$ dynamic range Logarithmic Detector. Datasheet available at: https://www.analog.com/media/en/technical-documentation/datasheets/AD8318.pdf [Accessed 15-09-2019].

[21] A. Arboleya, Y. Álvarez, F. Las-Heras, "Millimeter and Submillimeter Planar Measurement Setup," Proc. of the 2013 IEEE International Symposium on Antennas and Propagation. Orlando, USA, July 7-12, 2013. pp. 1-2.

[22] P. R. Rousseau, "Elemental probes for planar near-field antenna measurements," In Proc. of the 1999 IEEE Antennas and Propagation Society International Symposium, Orlando, USA, 11-16 July 1999, pp. 1802-1805.

[23] Numerical Electromagnetics Code (4NEC2). Available at: https://www.qsl.net/4nec2/ [Accessed 10-09-2019]. 